\section{The Achilles tendon}

\author{
Management of acute and \\ chronic conditions
}

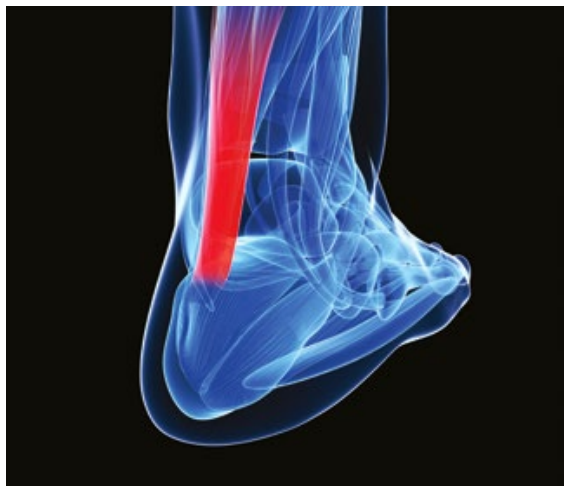

CPD

\section{Amy Touzell}

\section{Background}

The prevalence of acute and chronic conditions of the Achilles tendon is increasing among an ageing, active population. These conditions are a common cause of presentation to general practitioners and allied health practitioners. Achilles tendon injuries have a bimodal demographical presentation, with acute injuries commonly occurring in younger people and chronic conditions presenting in patients who are elderly.

\section{Objective}

The aims of this article are to discuss management of acute Achilles tendon ruptures in the primary care setting, explain the risks associated with calcaneal tuberosity fracture and discuss non-operative and surgical management of acute and chronic overload conditions of the Achilles tendon.

\section{Discussion}

Achilles tendon injuries can be divided into acute ruptures and chronic overuse injuries. Both can be debilitating, with significant morbidity for patients; fortunately, both types of injuries respond well to nonoperative interventions, with only a small proportion requiring surgery. Management of acute Achilles tendon rupture has evolved, with increasing evidence that nonoperative management is appropriate providing patients participate in a functional rehabilitation protocol. Chronic conditions such as the sequalae of an untreated rupture or Achilles tendinopathy can be debilitating but often respond well to non-operative management.
THE GASTROCNEMIUS AND SOLEUS MUSCLES, highly important for walking, running and impact activities, fuse to form the Achilles tendon, which is the largest and strongest tendon in the body. ${ }^{1}$ The tendon needs to withstand up to eight times body weight in force when undertaking sporting activities, ${ }^{2}$ and injuries to the Achilles tendon are common. Incidence and prevalence of Achilles tendon injuries has increased in the past 30 years, thought to be due to a combination of an active, ageing population and increased participation in sport and exercise programs in general. ${ }^{3}$

Achilles tendon injuries can be divided into acute ruptures and chronic overuse injuries. Both can be debilitating, with significant morbidity for patients; fortunately, both types of injuries respond well to non-operative and operative interventions, and only a small proportion requiring surgical intervention.

\section{Acute rupture}

Acute rupture of the Achilles tendon is a common injury, accounting for $20 \%$ of all large tendon ruptures. The estimated incidence ranges from 11 to 37 per 100,000 population, with men more than twice as likely to rupture their Achilles tendon than women. ${ }^{4}$ This injury has a bimodal distribution, with the first peak occurring in people aged $25-40$ years who are participating in high-energy sports, and the second peak in people aged $>60$ years. This second peak in more elderly patients is commonly associated with a low-energy injury, such as getting up from a seated position, after a history of Achilles pain. The risk of rerupture is higher in elderly patients with a degenerative tendon because of the inherent poor quality and diminished vascular supply of the tendon fibres. ${ }^{5}$ In the younger population, histological studies performed on acute Achilles tendon ruptures have shown clear degenerative changes despite little or no symptoms pre-injury. ${ }^{6}$

Patients who sustain an acute Achilles tendon rupture often describe a 'gunshot' sensation to the back of the leg. In the younger population with sports-associated Achilles tendon ruptures, up to $90 \%$ of cases are related to an acceleration-deceleration mechanism. ${ }^{7}$ Despite the 
characteristic history and symptoms, it is often a missed injury, with up to $25 \%$ of cases misdiagnosed at initial presentation. ${ }^{8}$

Fluoroquinolone antibiotics (commonly ciprofloxacin) have a known association with Achilles tendinitis and rupture, with a latency period of between two and 60 days. Patients aged $>60$ years are more likely to develop fluoroquinoloneinduced tendinitis. ${ }^{9}$ Other risk factors include rheumatoid arthritis, gout, ankylosing spondylitis, chronic uraemia and hyperparathyroidism, although these conditions are only responsible for Achilles tendon ruptures in $<2 \%$ of cases. ${ }^{1}$

To enable examination of a potential rupture of the Achilles tendon, the patient should be in the prone position or kneeling on a chair so the area can be easily inspected for bruising or other skin changes. The physical examination for detection of an acute Achilles tendon rupture has been described as 'Simmonds' triad': ${ }^{10}$

- Look at the angle of declination of the foot in comparison to the contralateral side.

- Feel for a palpable gap.

- Move ('squeeze test') - this involves a gentle squeeze of the calf with the foot hanging off the edge of the bed. If there is no movement of the foot, the test is positive, suggesting disruption of the gastrocnemius-soleus complex (Figure 1). The presence of two of the three signs has been shown to be $100 \%$ sensitive for an acute Achilles tendon rupture. ${ }^{11}$
Differential diagnoses that warrant exclusion include ankle fracture, ankle sprain or chronic tendinopathy to the long tendons at the back of the ankle. A careful history to ascertain the mechanism of injury combined with clinical examination can help exclude these injuries. It is important that an avulsion fracture of the calcaneal tuberosity (Figure 2) is not inadvertently diagnosed as an Achilles tendon rupture. These fractures commonly occur in elderly patients with diabetes, patients taking immunosuppressive medication or those with a history of osteopaenia. ${ }^{12}$ During examination, if palpation of the Achilles tendon yields a bony lump or there is evidence of skin tension, the patient should be placed in a plantarflexed cast and an urgent X-ray of the ankle ordered. This injury is a surgical emergency because of the risk of skin necrosis as the bony fragment causes tension on the overlying skin.

If there is any doubt regarding the diagnosis, ultrasonography of the Achilles tendon is useful to confirm examination findings. It is recommended that clinical findings are communicated to the reporting radiologist via a detailed referral form, specifying whether the concern is an acute or chronic condition, and patients are referred to centres with ultrasonographers and radiologists experienced in musculoskeletal ultrasonography. Careful correlation with clinical symptoms should be made, as chronic changes can be reported as a degenerative or partial tear of the tendon. It has been shown that ultrasonography is more effective than magnetic resonance imaging (MRI) for dynamically identifying the location of a tear or measuring the gap between the torn tendon edges, and differentiating between a partial and complete rupture. ${ }^{13}$ Therefore, MRI is not routinely used as a diagnostic tool for an acute rupture in this author's practice.

Management of acute Achilles tendon ruptures continues to be debated, but there is emerging evidence that supervised non-operative management results in very good outcomes. A randomised controlled trial of 144 patients randomised to operative and non-operative intervention reported no clinically important difference between groups with regard to strength, range of motion or calf circumference two years post-injury. ${ }^{14}$ The authors also reported a wound complication rate of $15 \%$ in the operative group. A Cochrane review reported a higher rerupture rate of $12 \%$ with non-operative treatment, compared with $5 \%$ in patients who receive surgery, but a complication rate of $30 \%$ in patients who receive surgery, compared with $8 \%$ in non-operatively treated patients. ${ }^{15}$

To reduce the risk of functional lengthening (in which the tendon heals in an elongated position causing long-term weakness), patients need to be placed in a

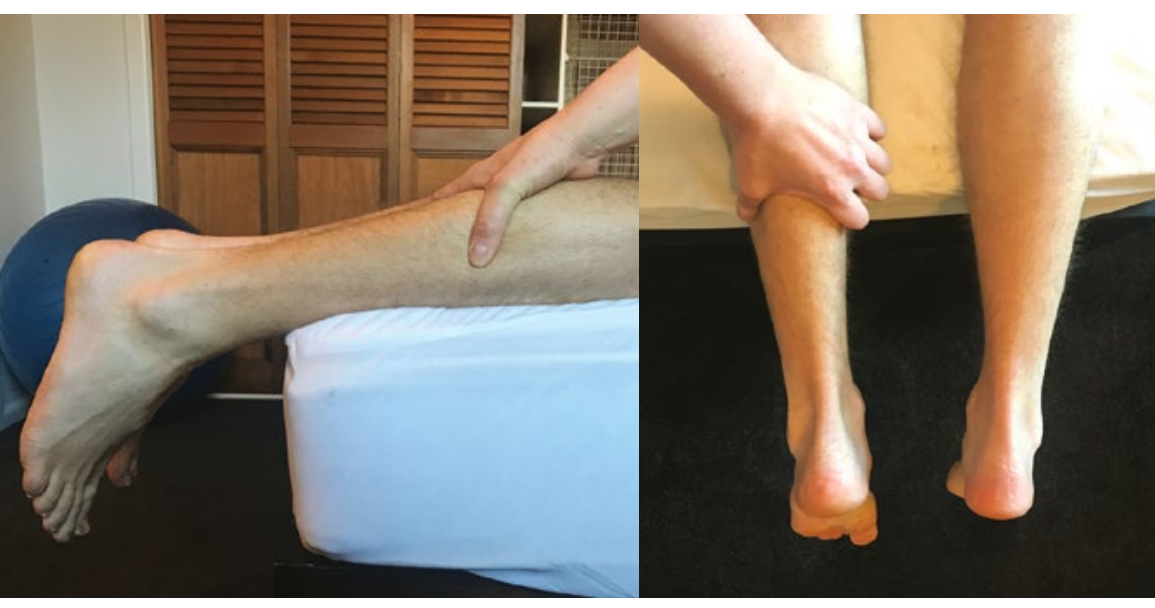

Figure 1. Demonstration of a negative Simmonds' squeeze test (no rupture evident)

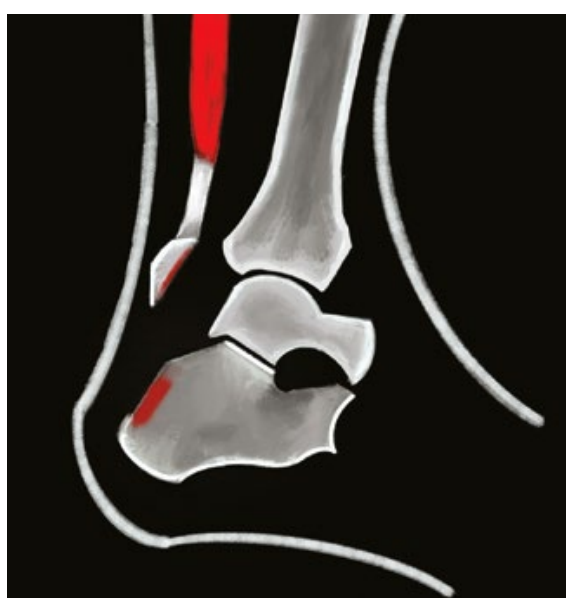

Figure 2. Calcaneal tuberosity fracture 
plantarflexed cast, backslab or removable orthosis with a heel wedge to force the ankle into plantarflexion as early as possible. ${ }^{5}$ Common removable orthoses include a controlled ankle motion (CAM) boot (Figure 3). Ideally the patient is placed in a cast or boot in the emergency department setting within hours of the injury occurring. A referral to the local public hospital fracture clinic or orthopaedic surgeon should be made as soon as possible after the injury so appropriate management options can be discussed with the patient. It is recommended that patients with a suspected acute Achilles tendon rupture are seen within seven days of the initial injury.

In this author's practice, patients are routinely treated non-operatively. An orthopaedic surgeon discusses the risk factors for operative and non-operative treatment modalities in detail with the patient, and patients are commenced on a functional rehabilitation program as directed by an Australian Physiotherapy Association-accredited sports physiotherapist or podiatrist employed by the practice. Involvement of an orthopaedic surgeon in non-operative management results in recognition of failure of non-operative measures as well as early discussion about potential surgery in the future if required. Rehabilitation protocols are shared with the patient's regular allied health practitioner for ongoing management, and they are reviewed by both surgeon and physiotherapist six weeks and 12 weeks post-injury.

It has been shown that a removable orthosis such as a CAM boot results in more favourable outcomes when compared with casting. ${ }^{16}$ It has also been shown that early weight-bearing and loading of the tendon result in improved clinical outcomes. ${ }^{17}$ Willets et al have published a detailed accelerated functional rehabilitation protocol for non-operative management of acute ruptures of the Achilles tendon, which is followed by this author's practice (Table 1 ). ${ }^{14}$ This protocol is well tolerated by patients, and the practice collaborates with local allied health practitioners (including physiotherapists and podiatrists) to improve compliance and supervision of non-operative management.
Tendinopathy and functional lengthening of the Achilles tendon

The term 'Achilles tendinopathy' encompasses a variety of pathologies characterised by pain and swelling in and around the Achilles tendon. Tendinopathy can have an acute or insidious onset. Achilles tendon overuse injuries are common in runners, with an annual incidence of between $7 \%$ and $9 \%$ in high-level runners. ${ }^{18}$ Notable short-term improvements have been seen with early referral to an experienced allied health practitioner such as a podiatrist or qualified sports physiotherapist. ${ }^{19}$ It may also be appropriate to refer patients to an exercise physiologist once a diagnosis is made, if a podiatrist or physiotherapist is not available, and many rural or regional exercise physiologists work in conjunction with a remote allied health team. Patients can access a chronic disease management plan under the Medicare Benefits Schedule if the condition has been present for $>6$ months. Most public hospital outpatient physiotherapy and podiatry departments also accept referrals for Achilles tendinopathy and related conditions. Early rehabilitation for chronic tendinopathy includes reduction in load (such as reducing running volume) and commencement of isometric exercises followed by strengthening exercises such as weighted calf raises. ${ }^{20}$

\section{Table 1. Accelerated functional rehabilitation protocol ${ }^{14}$}

\begin{tabular}{|c|c|}
\hline Phase & Instructions for patients \\
\hline Recovery phase & - Attend public fracture clinic or orthopaedic surgeon \\
\hline \multirow[t]{3}{*}{$0-2$ weeks post-injury } & $\begin{array}{l}\text { Wear plaster backslab in plantarflexion or boot with } 3 \mathrm{~cm} \\
\text { heel raise }\end{array}$ \\
\hline & - Commence early weight-bearing in boot with heel raise \\
\hline & - Use crutches for support \\
\hline Transition phase & - Remain in boot with heel raise \\
\hline \multirow[t]{5}{*}{ 2-6 weeks post-injury } & $\begin{array}{l}\text { Weight-bear as tolerated in boot and gradually wean off } \\
\text { crutches }\end{array}$ \\
\hline & - Perform hip and knee range-of-motion exercises in boot \\
\hline & - Perform static quadriceps and hamstring exercises in boot \\
\hline & - Wear boot while sleeping \\
\hline & - Use a single-leg exercise bike \\
\hline Return-to-activity phase & - Remove $1 \mathrm{~cm}$ heel wedge per fortnight \\
\hline \multirow[t]{3}{*}{ 6-10 weeks post-injury } & - Continue to weight-bear as tolerated in boot \\
\hline & $\begin{array}{l}\text { - If desired, remove boot for gentle non-weight-bearing } \\
\text { range-of-motion exercises of the ankle }\end{array}$ \\
\hline & $\begin{array}{l}\text { - If desired, commence swimming, avoiding pushing off the } \\
\text { wall and avoiding diving }\end{array}$ \\
\hline Prevention phase & - Remove heel lift \\
\hline \multirow[t]{4}{*}{ 10-12 weeks post-injury } & - Commence gentle dorsiflexion stretching \\
\hline & - Commence graduated resistance exercises \\
\hline & $\begin{array}{l}\text { - Commence cycling, elliptical machine, walking and } \\
\text { swimming }\end{array}$ \\
\hline & - Commence closed chain exercises and functional activities \\
\hline Active rehabilitation phase & - Remove boot \\
\hline \multirow{4}{*}{$\begin{array}{l}12 \text { weeks post-injury } \\
\text { and onwards }\end{array}$} & - Commence open chain exercises and functional activities \\
\hline & - Progress range of motion, strength and proprioception \\
\hline & - Commence plyometric training \\
\hline & - Commence sports-specific retraining \\
\hline
\end{tabular}


Patients may present in a delayed manner after an acute Achilles tendon rupture, sometimes months following the initial injury. A delay in presentation may be due to an individual patient not seeking medical care or misdiagnosis by the initial treating clinician. Management of a delayed presentation of an Achilles rupture and intractable Achilles tendinopathy are similar, and a referral to a sport and exercise physician (a recognised specialty in the Australian healthcare system) or orthopaedic surgeon is appropriate. Injections of steroid or platelet-rich plasma have a limited evidence base. ${ }^{21}$ Failing six months of non-operative treatment, surgery may be considered. Transfer of the flexor hallucis longus tendon is reserved for severe cases of Achilles tendinopathy, the development of functional lengthening after a rupture or delayed presentation after an acute rupture. ${ }^{22}$

\section{Insertional Achilles tendinopathy} Insertional Achilles tendinopathy is often a combination of retrocalcaneal bursitis, symptomatic Haglund's deformity and

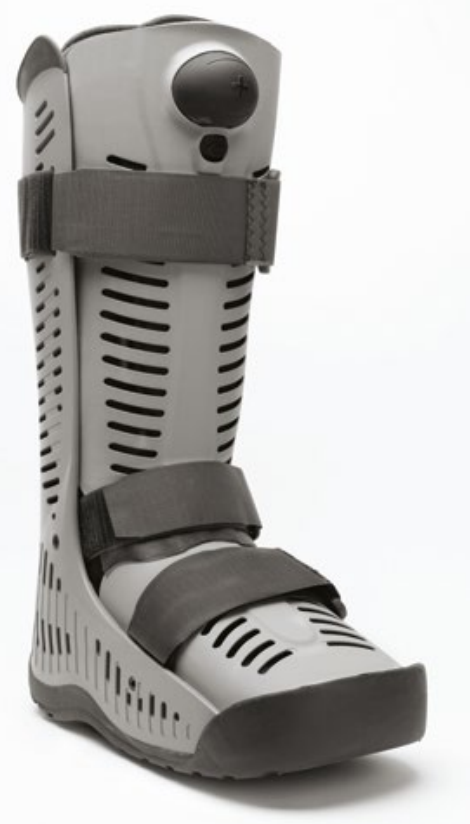

Figure 3. Controlled ankle motion (CAM) boot Source: Össur. All rights reserved. calcific deposits within the substance of the Achilles tendon close to the insertion point of the calcaneum. Chronic tightness of the Achilles tendon or calf musculature results in traction at the calcaneum, pulling the tendon insertion into the calcaneum. ${ }^{23}$

A Haglund's deformity is a bony prominence seen on the posterosuperior aspect of the calcaneal tuberosity (Figure 4). A tight Achilles tendon can rub on the prominent bone, causing an inflamed retrocalcaneal bursa. This presents as heel pain, exacerbated by physical activity such as walking or running. Patients report pain from direct palpation of the calcaneal tuberosity. X-ray and ultrasonography can show calcific deposits within the Achilles tendon as well as a Haglund's deformity.

Non-operative management can be as simple as avoidance of shoes that place pressure on the heel. Podiatry referral may be warranted for patients requiring adjustment of specific work shoes such as steel-capped boots. Rehabilitation, provided by an experienced allied health practitioner such as a podiatrist or physiotherapist, and nonsteroidal anti-inflammatory drugs are helpful in the acute setting. Rehabilitation involves addressing the contracted Achilles tendon and calf, reducing compression on the inflamed tendon then starting a strengthening program once acute inflammation settles. Patients may also benefit from a small heel lift in the shoe, or wearing shoes with a slight heel to reduce compression on the tendon. ${ }^{24}$
For cases refractory to non-operative management, surgery can be beneficial. This involves an open debridement of the Achilles tendon, excision of the Haglund's deformity and removal of the inflamed retrocalcaneal bursa (Figure 4).

\section{Deep vein thrombosis and pulmonary embolus}

A common complication of acute ruptures of the Achilles tendon or associated surgery is deep vein thrombosis (DVT). The rate of DVT in acute ruptures and surgery for chronic conditions has been reported as $2.67 \%,{ }^{25,26}$ and the rate of pulmonary embolus has been reported as $1.77 \% .{ }^{26}$ The clinician needs to be wary of the elevated risk of DVT in this population, particularly after a period of immobilisation. Routine use of chemoprophylaxis for DVT prevention is not indicated after acute rupture unless the patient has a specific thrombophlebotic condition such as Factor V Leiden disease. ${ }^{27}$ However, patients are encouraged to weight-bear when possible and are educated about re-presentation in the setting of calf pain, shortness of breath or chest pain.

\section{Summary}

Acute and chronic conditions of the Achilles tendon are a common presentation. For acute ruptures, immobilisation in plantarflexion helps
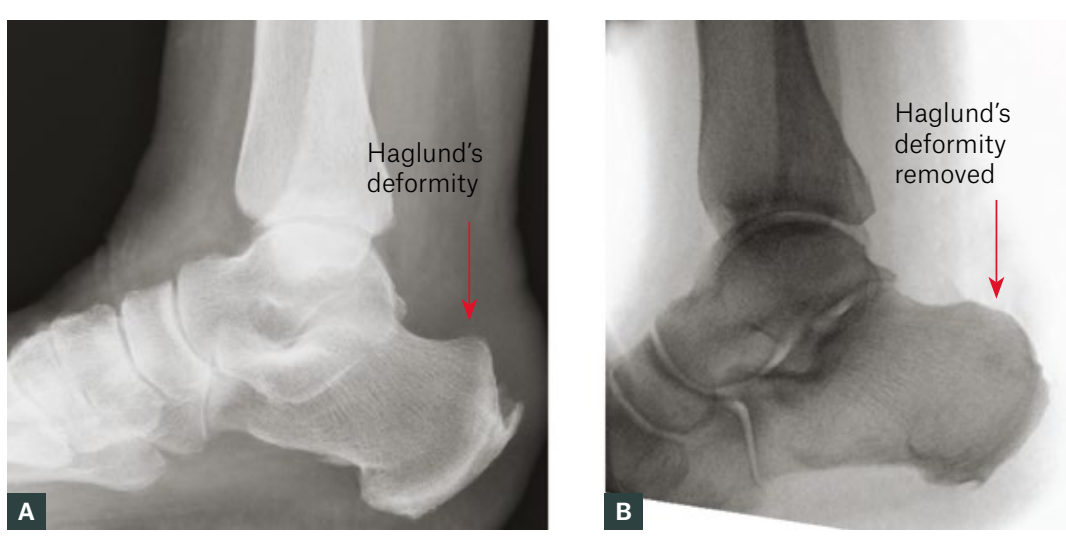

Figure 4. A. Haglund's deformity; B. Surgical removal of Haglund's deformity 
facilitate successful non-operative management when functional rehabilitation protocols are followed. Chronic tendinopathies, functional lengthening or rerupture usually can be treated with non-operative management. For refractory cases, a flexor hallucis longus tendon transfer is an effective, reliable procedure for chronic pathology of the Achilles tendon. Clinicians need to be wary of the elevated risk of DVT and pulmonary embolus in the acute and post-operative setting.

\section{Key points}

- Acute ruptures of the Achilles tendon can be successfully managed non-operatively.

- Chronic conditions respond well to rehabilitation in the majority of cases.

- A multidisciplinary approach to management is encouraged.

\section{Author}

Amy Touzell BMed, BMedSci (Hons), MPH (Dist), GradDipSurgAnat, FRACS (Orth), Senior Adjunct Lecturer, Peninsula Clinical School, Monash University, Vic; Visiting Medical Officer, Peninsula Health, Vic; Orthopaedic Surgeon, Holmesglen Private Hospital, Cabrini Private Hospital, Frankston Private Hospital, Vic.admin@southeastortho.com.au Competing interests: None.

Funding: None.

Provenance and peer review: Commissioned, externally peer reviewed.

\section{Acknowledgements}

The author would like to thank Mr Josh Ferguson, APAM, Sports and Exercise Physiotherapist, and Dr Alicia James PhD, APodA, Credentialed Paediatric Podiatrist and Head of Podiatry, Peninsula Health.

\section{References}

1. Maffulli N. Current concepts review - Rupture of the Achilles tendon. J Bone Joint Surg Am 1999;81(7):1019-36.

2. Järvinen TAH, Kannus P, Maffulli N, Khan KM. Achilles tendon disorders: Etiology and epidemiology. Foot Ankle Clin 2005;10(2):255-66 doi: 10.1016/j.fcl.2005.01.013.

3. Molloy A, Wood EV. Complications of the treatment of Achilles tendon ruptures. Foot Ankle Clin 2009;14(4):745-59. doi: 10.1016/j. fcl.2009.07.004

4. Park SH, Lee HS, Young KW, Seo SG. Treatment of acute Achilles tendon rupture. Clin Orthop Surg 2020;12(1):1-8. doi: 10.4055/cios.2020.12.1.1.

5. Gillissen S, Halperin I, Balesar V, Gorter E, Greeven A. Functional and early weightbearing protocol for Achilles tendon ruptures: A retrospective study. Eur J Trauma Emerg Surg 2020. doi: 10.1007/s00068-020-01376-3. [ePub ahead of print]
6. Young JS, Maffulli N. Etiology and epidemiology of Achilles tendon problems. In: Maffulli N, Almekinders LC, editors. The Achilles Tendon. London, UK: Springer-Verlag, 2007; p. 39-49.

7. Sharma P, Maffulli N. Tendon injury and tendinopathy: Healing and repair. J Bone Joint Surg Am 2005;87(1):187-202. doi: 10.2106/ JBJS.D.01850.

8. Inglis AE, Sculco TP. Surgical repair of ruptures of the tendo Achillis. Clin Orthop Relat Res 1981;(156):1960-69.

9. Levine AM, DiBona JR. Fluoroquinolones J Am Acad Orthop Surg 2002;10(1):1-4 doi: 10.5435/00124635-200201000-00001.

10. Singh D. Acute Achilles tendon rupture. Br J Sports Med 2017;51(15):1158-60. doi: 10.1136/ bjsports-2016-h4722rep.

11. Maffulli N. The clinical diagnosis of subcutaneous tear of the Achilles tendon. A prospective study in 174 patients. Am J Sports Med 1998;26(2):266-70. doi: 10.1177/03635465980260021801.

12. Rauer $T$, Twerenbold R, Flückiger R, Neuhaus $V$. Avulsion fracture of the calcaneal tuberosity: Case report and literature review. J Foot Ankle Surg 2018;57(1):191-95. doi: 10.1053/j.jfas.2017.07.016.

13. Maffulli N, Dymond NP, Regine R. Surgical repair of ruptured Achilles tendon in sportsmen and sedentary patients: A longitudinal ultrasound assessment. Int J Sports Med 1990;11(1):78-84. doi: 10.1055/s-2007-1024767.

14. Willits K, Amendola A, Bryant D, et al. Operative versus nonoperative treatment of acute Achilles tendon ruptures: A multicenter randomized trial using accelerated functional rehabilitation. J Bone Joint Surg Am 2010;92(17):2767-75. doi: 10.2106/ JBJS.I.01401.

15. Jones MP, Khan RJ, Carey Smith RL. Surgical interventions for treating acute Achilles tendon rupture: Key findings from a recent Cochrane review. 2012;94(12):e88. doi: 10.2106/JBJS.J.01829.

16. Nilsson-Helander K, Grävare Silbernagel K, Thomeé $\mathrm{R}$, et al. Acute Achilles tendon rupture: A randomized, controlled study comparing surgical and nonsurgical treatments using validated outcome measures. Am J Sports Med 2010;38(11):2186-93. doi: 10.1177/0363546510376052

17. Suchak AA, Bostick GP, Beaupré LA, Durand DC, Jomha NM. The influence of early weight-bearing compared with non-weight-bearing after surgical repair of the Achilles tendon. J Bone Joint Surg Am 2008;90(9):1876-83. doi: 10.2106/JBJS.G.01242.

18. Hreljac A. Impact and overuse injuries in runners. Med Sci Sports Exerc 2004;36(5):845-49. doi: 10.1249/01.mss.0000126803.66636.dd.

19. Alfredson H, Pietilä T, Jonsson P, Lorentzon R Heavy-load eccentric calf muscle training for the treatment of chronic Achilles tendinosis. Am J Sports Med 1998;26(3):360-66. doi: 10.1177/03635465980260030301.

20. Cook JL, Purdam CR. The challenge of managing tendinopathy in competing athletes. $\mathrm{Br} \mathrm{J}$ Sports Med 2014;48(7):506-09. doi: 10.1136/ bjsports-2012-092078.

21. de Vos RJ, Weir A, Tol JL, Verhaar JA Weinans $H$, van Schie HT. No effects of PRP on ultrasonographic tendon structure and neovascularisation in chronic midportion Achilles tendinopathy. Br J Sports Med 2011;45(5):387-92 doi: 10.1136/bjsm.2010.076398.

22. Gabel S, Manoli A 2nd. Neglected ruptures of the Achilles tendon. Foot Ankle Int 1994;15(9):512-17. doi: 10.1177/107110079401500912.

23. Saltzman CL, Tearse DS. Achilles tendon injuries. J Am Acad Orthop Surg 1998;6(5):316-25. doi: 10.5435/00124635-199809000-00007.
24. Reddy SS, Pedowitz DI, Parekh SG, Omar IM, Wapner KL. Surgical treatment for chronic disease and disorders of the achilles tendon. J Am Acad Orthop Surg 2009;17(1):3-14 doi: 10.5435/00124635-200901000-00002.

25. Wu Y, Mu Y, Yin L, Wang Z, Liu W, Wan H. Complications in the management of acute Achilles tendon rupture: A systematic review and network meta-analysis of 2060 patients. Am J Sports Med 2019;47(9):2251-60. doi: 10.1177/0363546518824601.

26. Bullock MJ, DeCarbo WT, Hofbauer MH, Thun JD. Repair of chronic Achilles ruptures has a high incidence of venous thromboembolism. Foot Ankle Spec 2017;10(5):415-20. doi: 10.1177/1938640016679706.

27. Patel A, Ogawa B, Charlton T, Thordarson D. Incidence of deep vein thrombosis and pulmonary embolism after Achilles tendon rupture. Clin Orthop Relat Res 2012;470(1):270-74. doi: 10.1007/ s11999-011-2166-6. 